\title{
Scalable Spatial Superresolution Using Entangled Photons
}

\author{
Lee A. Rozema, ${ }^{1, *}$ James D. Bateman, ${ }^{1}$ Dylan H. Mahler, ${ }^{1}$ Ryo Okamoto, ${ }^{1,2,3}$ Amir Feizpour, ${ }^{1}$ \\ Alex Hayat, ${ }^{1,4,5}$ and Aephraim M. Steinberg ${ }^{1,5}$ \\ ${ }^{1}$ Centre for Quantum Information \& Quantum Control and Institute for Optical Sciences, Department of Physics, \\ University of Toronto, 60 Saint George Street, Toronto, Ontario M5S 1A7, Canada \\ ${ }^{2}$ Research Institute for Electronic Science, Hokkaido University, Kita-ku, Sapporo 060-0812, Japan \\ ${ }^{3}$ The Institute of Scientific and Industrial Research, Osaka University, Mihogaoka 8-1, Ibaraki, Osaka 567-0047, Japan \\ ${ }^{4}$ Department of Electrical Engineering, Technion, Haifa 32000, Israel \\ ${ }^{5}$ Canadian Institute for Advanced Research, Toronto, Ontario M5G 1Z8, Canada
}

(Received 6 December 2013; published 2 June 2014)

\begin{abstract}
N00N states-maximally path-entangled states of $N$ photons-exhibit spatial interference patterns sharper than any classical interference pattern. This is known as superresolution. However, even given perfectly efficient number-resolving detectors, the detection efficiency of all previous measurements of such interference would decrease exponentially with the number of photons in the N00N state, often leading to the conclusion that $\mathrm{N} 00 \mathrm{~N}$ states are unsuitable for spatial measurements. A technique known as the "optical centroid measurement" has been proposed to solve this and has been experimentally verified for photon pairs; here we present the first extension beyond two photons, measuring the superresolution fringes of two-, three-, and four-photon N00N states. Moreover, we compare the N00N-state interference to the corresponding classical superresolution interference. Although both provide the same increase in spatial frequency, the visibility of the classical fringes decreases exponentially with the number of detected photons. Our work represents an essential step forward for quantum-enhanced measurements, overcoming what was believed to be a fundamental challenge to quantum metrology.
\end{abstract}

Many essential techniques in modern science and technology, from precise position sensing to high-resolution imaging to nanolithography, rely on the creation and detection of the finest possible spatial interference fringes using light. Classically, all such measurements face a fundamental barrier related to the "diffraction limit," which is determined by the wavelength of the light [1], but quantum entanglement can be used to surpass this limit by making the spatial interference fringes sharper (a result referred to as superresolution) [2,3]. In particular, the $\mathrm{N}$-photon entangled "N00N" state can display an $\mathrm{N}$-photon interference pattern $N$ times finer than that of classical light $[4,5]$. However, NOON states suffer from a weakness that has made their advantage controversial: the probability of all $N$ photons arriving at the same place, and thus the detection efficiency, decreases exponentially with $N[6,7]$. Here we implement the optical centroid measurement (OCM) proposed by Tsang [8] to overcome this problem. A proof-of-principle experiment confirming the underlying concept of the OCM was recently performed [9], but, being limited to only two photons and two "movable" detectors, it could not probe the scaling properties of the OCM. In our experiment, using an array of 11 fixed detectors, we measure two-, three-, and four-photon spatial fringes, and find that their visibility does not detoriate with the number of entangled photons. The visibility of an unentangled OCM, on the other hand, decays exponentially. Our results show that, given more efficient detectors, the OCM could potentially be scaled to high photon numbers and perhaps provide a means for practical quantum imaging. Our experiment also represents the highest spatial superresolution to date [10-12].

In 2000, Boto et al. pointed out that entangled states of light offer a way to improve the resolution of interferometers beyond the diffraction limit, which determines the smallest spatial features achievable in classical optical systems [2]. This limit is set by the fringe spacing of the interference pattern created by two beams of wavelength $\lambda$ meeting at an angle $\theta$, which is $\lambda /(2 \sin (\theta / 2))$. Under no conditions can a spacing smaller than $\lambda / 2$ be attained classically. Boto et al. circumvented this classical limit by introducing the entangled NOON state, an equal superposition of all $\mathrm{N}$ photons in mode $\vec{k}_{1}$ and all $N$ photons in mode $\vec{k}_{2}:\left|\psi_{N}\right\rangle=\frac{1}{\sqrt{2}}\left(|N, 0\rangle_{\vec{k}_{1}, \vec{k}_{2}}+|0, N\rangle_{\vec{k}_{1}, \vec{k}_{2}}\right)$.

If $\vec{k}_{1}$ and $\vec{k}_{2}$ are two spatial modes which interfere at a detection plane [Fig. 1(a)], the probability of detecting all of the photons at a given position will display spatial fringes with a period of $\lambda /(2 N \sin (\theta / 2))$, where $\theta$ is the angle between $\vec{k}_{1}$ and $\vec{k}_{2}$ (assuming $\left|\vec{k}_{1}\right|=\left|\vec{k}_{2}\right|=(2 \pi / \lambda)$ ). The period of the NOON fringes is $\mathrm{N}$ times smaller than that of classical fringes, suggesting that NOON states could be used to increase the resolution of optical systems by a factor of $N$. This observation has led to much subsequent work on N00N states [4,5,13-16] and their application in tasks such 


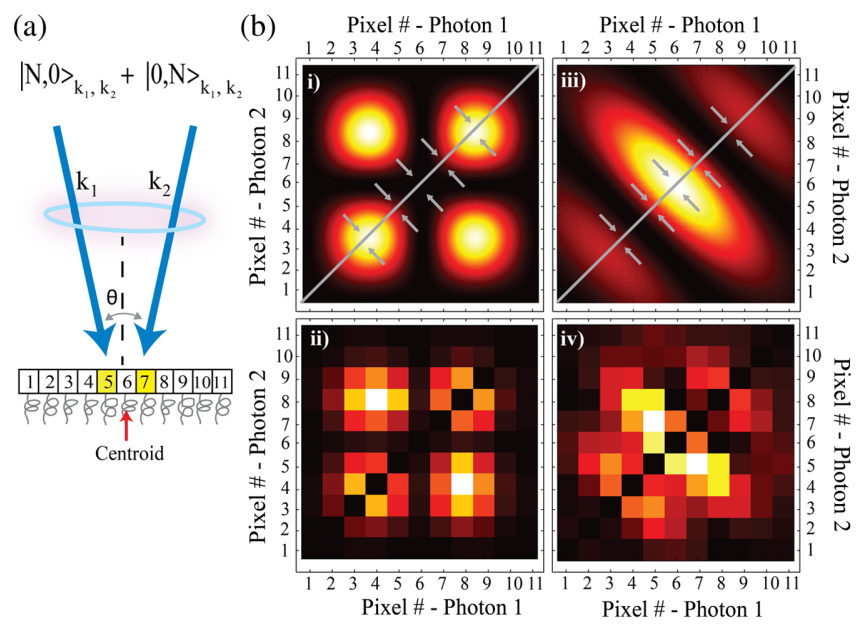

FIG. 1 (color online). The Centroid measurement: (a) Schematic of the detection scheme. Light from two modes, $\vec{k}_{1}$ and $\vec{k}_{2}$, is incident on an array of single photon detectors. Correlations between all the detectors are measured and recorded. (b) Twophoton joint-probability functions. (i) and (iii) are theoretical two-photon joint-probability functions when classical light and $N=2$ N00N states illuminate the detector array, respectively. (ii) and (iv) are the experimental two-photon joint-probability functions. Since there is no way to distinguish photon 1 from photon 2 , we could only measure the half of the plot where $x_{1}>x_{2}$; in plots (ii) and (iv) we have mirrored this data about the diagonal $\left(x_{1}=x_{2}\right)$ for comparison to theory. The diagonal region is dark since two photons arriving simultaneously at the same SPCM cannot both be detected.

as quantum lithography and quantum imaging $[10-12,17]$. However, the individual photons in the NOON state cannot be localized to better than $\lambda / 2$, regardless of the narrow spatial scale of the $N$-photon correlation fringes [6,7]. This means that the probability of a given photon landing within some small region of size $r$ will always be $\lesssim 2 r / \lambda$, and that the probability for all $N$ photons to arrive at the same region is $\lesssim(2 r / \lambda)^{N}$. Thus even with perfect detectors the efficiency of such a detection scheme decreases exponentially with $N$, leading to the conclusion that N00N states are of little practical use for applications requiring spatial interference. The OCM was proposed to address this problem, resurrecting the hope of applying such states to high-resolution position measurements [8].

The OCM displays $N$-fold superresolution without requiring all the photons to arrive at the same point in space. Instead, it keeps track of every $N$-photon event, regardless of which combination of detectors fires. By using appropriate post-processing, the OCM nevertheless unveils the $N$-photon quantum interference. For simplicity, consider interfering a two-photon N00N state on an array of photon detectors [Fig. 1(a)]. Most of the time, two different detectors fire; occasionally, both photons reach the same detector. The original proposals only retained those rare events in which a single detector registered both photons, observing that this rate exhibited sub-wavelength fringes as a function of detector position. By contrast, the OCM keeps all the events, recording the "centroid," or the average of the detected positions of the photons; remarkably, subwavelength fringes are also observed as a function of this centroid, obviating the need to discard the bulk of the events [8].

A more visual way to look at the OCM is illustrated in Fig. 1(b), which shows plots of the joint probabilities for photon 1 to arrive at pixel $x_{1}$ and photon 2 to arrive at pixel $x_{2}$ in coincidence. In a two-photon-absorber measurement, an event is only registered if $x_{1}=x_{2}$ (both photons arrive at the same point). The resulting signal will be given by the photon correlations along the grey diagonal lines drawn in (i) (for classical light) and (iii) (for a N00N state). This also means that all of the other possible events are discarded. The OCM signal utilizes all of this data. For two photons, the OCM signal can be visualized as the integral of the joint-probability functions onto the grey diagonal lines drawn in Fig. 1(b) (i) and (iii). In other words, it is a histogram of all the points plotted versus their centroid position, $\left(x_{1}+x_{2}\right) / 2$. Notice that the OCM also increases the number of "effective pixels" by a factor of $N$, since if, for example, detectors 3 and 4 fire the value of the centroid is 3.5, making it possible to detect superresolution features which are much smaller than the physical pixel size. For higher values of $N$, the $N$-photon centroid is computed similarly from an $N$-photon joint-probability function [18]. Unlike the $N$-photon-absorbing proposal, which keeps only $O\left(1 / D^{N-1}\right)$ of the $N$-photon events (if there are $D$ pixels), this method uses them all. Note that even though the OCM provides an efficiency enhancement of $D^{N-1}$, if one photon is detected with probability $\eta$ then $N$ photons will be detected with probability $\eta^{N}$, regardless of the detection scheme.

From Fig. 1(b), it can be seen that performing an OCM on a NOON state will result in a signal with the same periodicity as the $\mathrm{N}$-photon absorption detection scheme. It is also evident that performing the OCM on classical light will result in fringes with this enhanced periodicity. Although the classical OCM indeed results in a signal with a period of $\lambda /(2 N \sin \theta)$, its visibility decreases exponentially with $N$, as $V_{c}=\left(1 / 2^{N-1}\right)$. (As shown in the Supplemental Material [19], this is because performing an $N$-fold OCM on classical light results in signal which is the convolution of the singles intensity pattern with itself $N$ times.) However, as we show experimentally for two-, three-, and four-photon N00N states, the visibility of the NOON state OCM remains constant, independent of $N$.

Experimentally, we produce N00N states in polarization using a technique which, given bright down-conversion sources, can produce N00N states of arbitrary $N[15,16]$. Our source [Fig. 2(a)] creates N00N states by combining laser light with light from a type-I collinear downconversion (DC) source. The DC light is generated by pumping a $1 \mathrm{~mm}$ BBO crystal, cut for type-I phase 

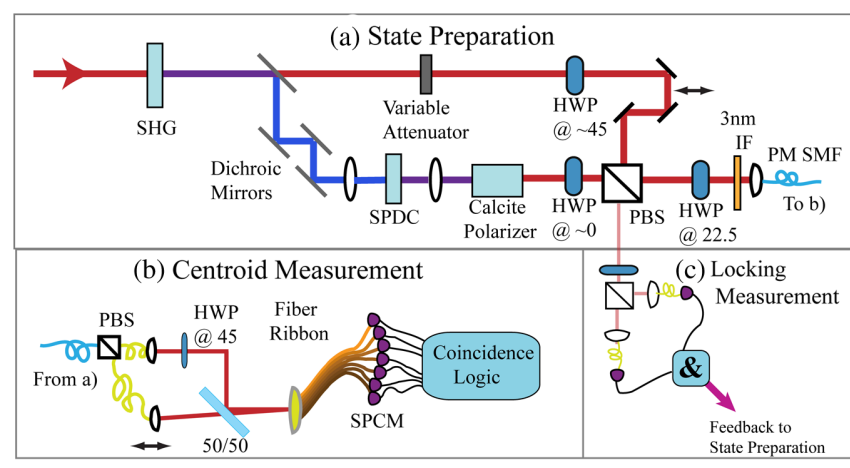

FIG. 2 (color online). Schematic of the experimental apparatus: (a) N00N state preparation. Laser light and down-converted light are combined to create polarization N00N states for a range of different values of $N$. (b) Centroid measurement. The polarization N00N states are converted into path-entangled N00N states and interfered on a multimode fiber ribbon connected to 11 singlephoton counting modules (SPCM). (c) Locking measurement. A small amount of down-converted and laser light is sent into the "locking port" and used to measure any phase drift between the laser and down-conversion paths.

matching, with $600 \mathrm{~mW}$ average power of $404 \mathrm{~nm}$ light. The $404 \mathrm{~nm}$ pump is generated by frequency-doubling light from a femtosecond Ti:Sapph laser (running at $1.4 \mathrm{~W}$ average power and $76 \mathrm{MHz}$ repetition rate, and centered at $808 \mathrm{~nm}$ ) using a $2 \mathrm{~mm}$ long crystal of BBO. We measure approximately 12,000 two-photon counts/s from the DC source, with a coupling efficiency (pairs/singles) of $9.5 \%$ when single-photon detectors are placed directly after the in-fiber polarizing beam splitter (PBS) of panel b. The DC light is spatially overlapped with light from the Ti:Sapph laser at a PBS, passed through a half wave plate at $22.5^{\circ}$, and coupled into polarization-maintaining single-mode fiber. To generate N00N states, the relative phase between the two arms must be set to zero, and the relative amplitudes balanced. The source is optimized for NOON states of different $\mathrm{N}$ by simply changing the relative amplitude between the laser and DC light [15]. Ideally, two- and three-photon N00N states can be made perfectly by setting the two-photon rate from the laser equal to that from the DC light (which we will refer to as "configuration 1"). Although our source cannot make perfect four-photon N00N states, it can, in principle, make a state which has 93\% fidelity with the ideal four-photon N00N state (and an OCM visibility of 93\%). This requires that the laser twophoton rate be three times larger than the DC two-photon rate. However, even if the laser rate is further increased, increasing the four-photon count rate, the fidelity of the four-photon state will not be significantly degraded [20]. To make four-photon N00N states, we choose to make the laser two-photon rate 8.5 times larger than the DC rate (configuration 2), which leads to a fidelity with the ideal state of $85 \%$, a theoretical OCM visibility of $85 \%$, and increases the four-photon rate by about a factor of 10 (compared to the configuration in which the four-photon fidelity is optimized).

The phase between the two arms must also be stabilized to make N00N states. We accomplished this by using a piezoelectric-driven trombone arm in the laser path. To generate a feedback signal, small amounts of DC and laser light are sent through the other port of the state-preparation PBS, to the "locking measurement" [Fig. 2(c)]. We measure 500 down-converted two-photon counts/s at the locking measurement and 5000 two-photon counts/s from the laser, creating a low-fidelity two-photon N00N state. This state will be phase shifted if the phase between the two arms drifts, and can therefore be used to track the phase drift. After acquiring counts for $5 \mathrm{~s}$, the phase of the state is measured, and any phase drift between the two arms is corrected. This allows us to keep the N00N-state source stable for days.

Once polarization N00N states are collected into polarization-maintaining single-mode fiber from the source, they are sent to the OCM apparatus [Fig. 2(b)]. There, they are converted into path-entangled NOON states using an in-fiber PBS. Once the output polarizations are matched, the two modes are spatially interfered, by overlapping them at a 50:50 beam splitter, at an angle of $\theta=0.16 \mathrm{mrad}$. These overlapped modes are focused onto a "fiber-ribbon" (serving as our fixed-detector array) with cylindrical lenses. The visibility of the interference pattern formed across the detector array is measured to be $90 \%$ using classical light. The phase of this interference signal is actively locked by observing the phase on the detector array, and feeding this back to a piezoelectric actuator which moves one of the collimators.

Our array of single-photon detectors consists of 12 multimode fibers, mounted in a standard MTP12 fiber ribbon. The fiber cores are $62.5 \mu \mathrm{mm}$ in diameter, and are mounted linearly with a $250 \mu \mathrm{m}$ separation between fiber centers. This results in a fill factor (and therefore maximum coupling efficiency) of $\approx 25 \%$. Eleven of these fibers are coupled to Perkin-Elmer single-photon counting modules (SPCM), which are all connected to a custom-built FPGAbased coincidence counting circuit capable of detecting coincidence events among all possible detector combinations. Using classical light, we measure an overall coupling efficiency of $20 \%$ into the 11 fibers making up our detector array. The additional losses in the OCM apparatus further reduces the final coupling efficiency of the DC photons (pairs/singles) from $9.5 \%$ to $0.2 \%$ (measured in the fiber ribbon by summing the two-photon counts, and dividing by the sum of the singles).

We run the N00N-state source in the two configurations (described above) to take data. In configuration 1, we observed 150 two-photon NO0N states per second and 328 three-photon N00N states in 66 minutes, using the fiber ribbon. In configuration 2, we measured 284 four-photon N00N states in 57 hours. The measurement time increases 
with $\mathrm{N}$ because we couple much less than one photon-pair per pulse, and a four-photon N00N state is created only when two pairs are emitted in a single pulse, which is even rarer. Recent progress towards on-demand sources of entangled photons [21,22] could greatly reduce the measurement time [16].

The resulting OCMs are plotted in the second column of Fig. 3 (circles). The three panels, from top to bottom, are the two-, three-, and four-photon OCMs. A sinusoid with a Gaussian envelope is fitted to the data (solid lines), and the visibility extracted from the sinusoidal part of the fit. We expect the OCM data have a Gaussian envelope narrowing with $N$; this is because the singles intensity has a Gaussian envelope and it becomes less likely to produce a centroid result far from the center as $N$ increases. In the fits to the two- to four-photon data, the period of the $N$-photon centroid is constrained to be the single-photon period divided by $N$. The resulting visibilities are $49 \pm 4 \%, 44 \pm$ $5 \%$ and $41 \pm 6 \%$, for the two-, three-, and four-photon OCMs respectively. The three- and four- photon visibilities are well in excess of the classical limits of $25 \%$ and $12.5 \%$, surpassing them by 3.6 and 4.7 standard deviations, respectively. To account for imperfections in the source (arising from imperfect coupling of the down-converted

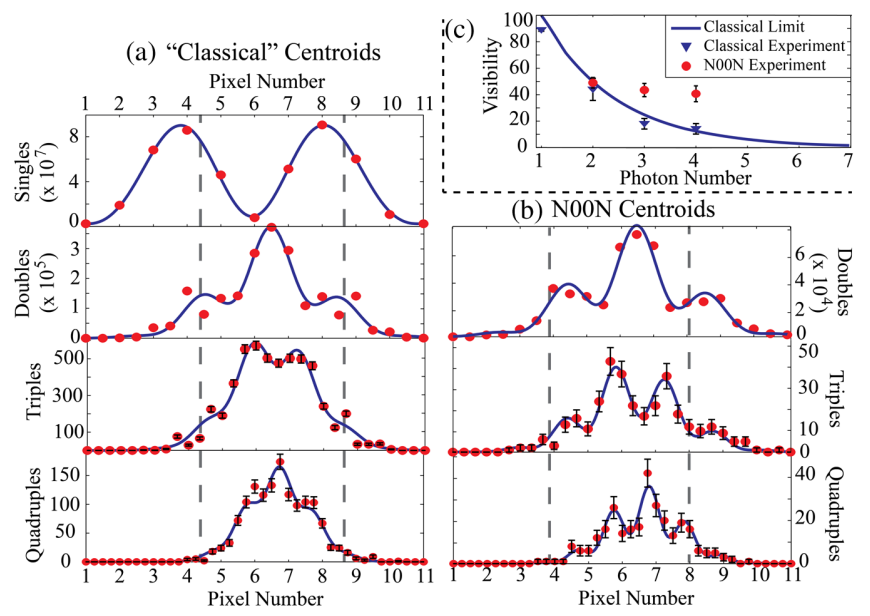

FIG. 3 (color online). (a),(b) Experimentally measured centroids: The first column (a) is the result of a centroid measurement performed on classical light, and the second column (b) the result for NOON states. The circles are the measured data, and the solid curves are fits from which the visibility is extracted. The total number of counts is plotted (acquisition times given in the text). The error bars are calculated assuming Poissonian counting statistics, and are not shown for the one- and two-photon data as they are much smaller than the circles. The vertical dashed lines indicate the period of the classical interference pattern. (c) Scaling of visibility with photon number: A plot of the visibility of the centroid pattern versus photon number. The blue curve is the visibility which is predicted for the ideal classical centroid measurement, the blue triangles are the experientially measured classical visibilities, and the red circles are the measured visibilities of the quantum optical centroid measurement. light), accidental counts were subtracted from the OCM data (explained in the Supplemental Material [19]). The OCM visibilities, after subtracting these accidental counts, are $65 \pm 4 \%, 61 \pm 6 \%$ and $59 \pm 8 \%$, for the two-, three-, and four-photon OCMs, respectively. The accidentalcorrected visibilities are better than the classical limits by 3.8, 6.0 and 5.8 standard deviations, respectively. For comparison, we experimentally perform an OCM on classical light (by blocking the DC light). These results are plotted in the first column of Fig. 3. The measurement times are 10 minutes for the one-, two-, and three-photon classical centroid measurements, and 45 minutes for the four-photon measurements. The 'classical' acquisition times are shorter than for the NOON states since the laser rate can be turned up much higher, as it does not need to match the down-converted rate. These visibilities are $44 \pm 9 \%$, $18 \pm 4 \%$, and $14 \pm 4 \%$, agreeing well with the expected exponential decay. The increase in the number of "effective pixels" is also evident in these data; the singles signal has 11 points, while the four-photon data have 44. In practical terms, this means an array with fixed spacing could be used to perform an OCM with a N00N state of arbitrary $N$, unlike the standard $N$-photon-absorbing scheme which would require the pixel spacing to scale as $1 / N$.

To study the scalability of our experiment, we plot the various visibilities versus photon number in Fig. 3(c). The solid curve is the theoretical prediction for the visibility of the classical OCM signal. The experimentally measured classical visibilities (triangles) agree well with this curve, demonstrating a clear exponential decay. The visibility of the OCM signal performed on a NOON state should be constant (in principle it should be 1.0). Although the measured raw visibilities (circles) are not perfect, they are nearly independent of $N$ (this trend is also evident in the accidental-corrected visibilities). These measurements demonstrate a crucial element of the scalability of the OCM-the N00N visibilities remain constant while the classical visibilities decrease exponentially. The OCM's detection efficiency enhancement is the other essential aspect of its scalability. One could directly measure this enhancement by comparing the rate of an $N$-photon absorber measurement to our OCM rate. However, we do not this since it would require replacing each of our 11 SPCMs with a number-resolving detector. This points to another benefit of the OCM: we were able to measure four-photon quantum interference without the need for number-resolving detectors or a four-photon absorber. Our experiment indicates that, as technology progresses, using high-efficiency detector arrays and on-demand entangledphotons sources, our techniques could be used to measure high-NOON superresolution signals with high visibility.

In conclusion, we have measured spatial interference patterns of two-, three-, and four-photon N00N states using an 11-detector multiplexed measurement (the OCM). Our N00N-state source produces high-fidelity, high- $N$ NOON 
states $[15,16,20]$, and we have shown that the NOON state OCM signal displays $N$-fold superresolution, and a visibility that is nearly independent of $N$. Thus, our implementation is naturally applicable to higher photon numbers. This could be achieved using several exciting new technologies which are continually advancing, such as high-efficiency single-photon detectors [23,24], high-fillfactor single-photon detector arrays [25], and brighter down-conversion sources with high coupling efficiency $[21,22]$. Our experiment demonstrates a practical implementation to overcome the problem of efficiently detecting $\mathrm{N}$-photon states-a fundamental challenge to practical quantum metrology - and could open the door for Heisenberg-limited phase detection and quantum imaging.

We thank Amr Helmy and Peter Herman for suggesting the use of a fiber ribbon. We are very thankful for Alan Stummer's work on designing and building our 11-channel coincidence circuit. We acknowledge support from NSERC and CIFAR. R. O. acknowledges additional support from the Yamada Science Foundation. L. A. R. and J.D. B. contributed equally to this work.

*Corresponding author. lrozema@physics.utoronto.ca

[1] L. Rayleigh, Philos. Mag. 8, 261 (1879).

[2] A. N. Boto, P. Kok, D. S. Abrams, S. L. Braunstein, C. P. Williams, and J. P. Dowling, Phys. Rev. Lett. 85, 2733 (2000).

[3] P. Kok, A. N. Boto, D. S. Abrams, C.P. Williams, S. L. Braunstein, and J.P. Dowling, Phys. Rev. A 63, 063407 (2001).

[4] M. W. Mitchell, J. S. Lundeen, and A. M. Steinberg, Nature (London) 429, 161 (2004)

[5] P. Walther, J.-W. Pan, M. Aspelmeyer, R. Ursin, S. Gasparoni, and A. Zeilinger, Nature (London) 429, 158 (2004).

[6] O. Steuernagel, J. Opt. B 6, S606 (2004).
[7] M. Tsang, Phys. Rev. A 75, 043813 (2007).

[8] M. Tsang, Phys. Rev. Lett. 102, 253601 (2009).

[9] H. Shin, K. Wai Clifford Chan, H. J. Chang, and R. W. Boyd, Phys. Rev. Lett. 107, 083603 (2011).

[10] M. D’Angelo, M. V. Chekhova, and Y. Shih, Phys. Rev. Lett. 87, 013602 (2001).

[11] Y. Kawabe, H. Fujiwara, R. Okamoto, K. Sasaki, and S. Takeuchi, Opt. Express 15, 14244 (2007).

[12] Y.-S. Kim, O. Kwon, S. M. Lee, J.-C. Lee, H. Kim, S.-K. Choi, H. S. Park, and Y.-H. Kim, Opt. Express 19, 24957 (2011).

[13] T. Nagata, R. Okamoto, J. L. O'Brien, K. Sasaki, and S. Takeuchi, Science 316, 726 (2007).

[14] L. K. Shalm, R. B. A. Adamson, and A. M. Steinberg, Nature (London) 457, 67 (2009).

[15] H. F. Hofmann and T. Ono, Phys. Rev. A 76, 031806 (2007).

[16] I. Afek, O. Ambar, and Y. Silberberg, Science 328, 879 (2010).

[17] M. B. Nasr, B. E. A. Saleh, A. V. Sergienko, and M. C. Teich, Phys. Rev. Lett. 91, 083601 (2003).

[18] Q.-u.-A. Gulfam and J. Evers, Phys. Rev. A 87, 023804 (2013).

[19] See Supplemental Material at http://link.aps.org/ supplemental/10.1103/PhysRevLett.112.223602 for a derivation of the classical centroid visibility and for details on our estimation of the accidental count rate.

[20] S. Rosen, I. Afek, Y. Israel, O. Ambar, and Y. Silberberg, Phys. Rev. Lett. 109, 103602 (2012).

[21] G. Harder, V. Ansari, B. Brecht, T. Dirmeier, C. Marquardt, and C. Silberhorn, Opt. Express 21, 13975 (2013).

[22] A. Predojević, S. Grabher, and G. Weihs, Opt. Express 20, 25022 (2012).

[23] D. Rosenberg, A. E. Lita, A. J. Miller, and S. W. Nam, Phys. Rev. A 71, 061803 (2005).

[24] A. E. Lita, A. J. Miller, and S. W. Nam, Opt. Express 16, 3032 (2008).

[25] M. Ghioni, A. Gulinatti, I. Rech, F. Zappa, and S. Cova, IEEE J. Sel. Top. Quantum Electron. 13, 852 (2007). 